\title{
SECTOR SYNTHESIS OF ANTENNA ARRAY USING GENETIC ALGORITHM
} \author{
2 \\ Abdelaziz A. Abdelaziz and Hanan A. Kamal \\ 'Assoc. Prof., Department of Electrical Engineering, Faculty of Engineering, \\ Misr University for Science and Technology (MUST), Cairo, Egypt \\ Asstt Prof., Department of Electrical Engineering, Faculty of Engineering, \\ Cairo University, Giza, Egypt \\ Email: abdelaziz abdelmonem@yahoo.com, hanan ak2003@yahoo.com
}

\begin{abstract}
This paper introduces a new application of genetic algorithm (GA) in dynamic sector synthesis of antenna array for mobile base transceiver station (BTS). The basic objective is to have narrow beam widths in heavy handoff areas and wider beam widths in areas with low traffic density. By predefining the beam widths and thus the beams of each sector, optimal sectoring can be achieved using GA at different time of the day as traffic changes. The proposed algorithm consists of the simple GA with new adaptive mutation mechanism. When compared with simple GA, the proposed algorithm shows faster convergence. The results obtained are more stable and having higher fitness.
\end{abstract}

\section{Keywords: Antenna Array (AA), Sector Synthesis (SS), Genetic Algorithm (GA)}

1. INTRODUCTION optimal sectoring can be achieved at different time of the day as traffic changes. Over the last few years, the number of subscribers The main objective of this work is to get the to wireless services has increased at an explosive optimal selection of the assigned sectors angles of rate. This growing demand for wireless the BTS such that the different sectors have communications services is constantly increasing properly balanced load all the time by using GA. the need for better coverage, improved capacity These sectors will be formed from different beams. and higher quality of service. These demands can Different beam angles can be obtained by be achieved by using smart antennas [1]. Smart changing the number of antenna array elements or antennas [2] are critical enabling technologies that the amplitude distribution they are fed. The first will provide high mobility in dense traffic areas. part of this work is to investigate the beam widths Smart antenna system [3], as one of the most produced using a linear array with varying number promising technologies in the cellular area, using of array elements and with different amplitude switched beam and adaptive antenna array with distributions. An example of BTS will be microstrip technology [4] are rapidly becoming an considered with options of 3 and 4 sectors. The integral part of both analog and digital cellular decision of sectors assignment depends on the networks. Using the approach of smart antenna traffic capacity which can be represented by the with switched beam antenna system [5], an measured power density received by the antennas intelligent sector synthesis of varying azimuth and of the BTS. The power measurement stage has not beam width can be established. Dynamic sectors been considered in our work but we will assume synthesis can be achieved by making the angles of two different power distributions for the assumed the sectors varying according to the traffic capacity 3 and 4 sectors with assigned hot spots. In the which varies from time to time during the day. By second part of this work, an adaptive GA is doing so, traffic load balancing can be achieved. introduced and used to solve the above problem. Another approach for load balancing is to use the This algorithm has a new adaptive mutation genetic algorithm $[6,7,8]$. The GA is a powerful mechanism which will maintain the best scheme in resource for electromagnetic optimization the solution and at the same time provides the problems [9]. Under GA control, the sector beam search space with new solution elements. The width can be varied with narrow beam widths in structure of the algorithm and a comparison heavy handoff areas (hot spots) and wider beam between the obtained results and previous work widths in areas with low traffic density. Thus, has been made. Results have proved the effectiveness of the proposed algorithm. 


\section{BEAM WIDTH INVESTIGATION}

The first part of this work is to investigate the beam widths produced using a linear array with varying number of array elements and with different amplitude distributions. The number of elements used in the simulation is in the range of 3 to 12 . The values are chosen such that they can represent a practical implementation. There are many famous linear array synthesis techniques have been used to produce shaped beam patterns [10]. Table 1 shows the simulated beam widths (BW) produced from a linear array elements of isotopic type with resonance frequency of $2 \mathrm{GHz}$ and inter-element spacing $\lambda / 2$. Simulation results are obtained using antenna design software PCAAD 4.0 [11] for the most famous amplitude distributions.

Table 1: The 3-dB beam widths (BW), in degrees, for different amplitude distributions and element numbers

\begin{tabular}{|l|l|l|l|l|l|l|}
\hline $\begin{array}{l}\text { Number of } \\
\text { elements }\end{array}$ & $\begin{array}{l}\text { BW for } \\
\text { Cheby. } \\
\text { Weight (1) }\end{array}$ & $\begin{array}{l}\text { BW for } \\
\text { Woodword } \\
\text { Weight (2) }\end{array}$ & $\begin{array}{l}\text { BW for } \\
\text { Uniform } \\
\text { Weight (3) }\end{array}$ & $\begin{array}{l}\text { BW for } \\
\text { Cosine } \\
\text { Weight (4) }\end{array}$ & $\begin{array}{l}\text { BW for } \\
\text { Taylor } \\
\text { Weight } \\
(5)\end{array}$ & $\begin{array}{l}\text { BW for } \\
\text { Binom. } \\
\text { Weight (6) }\end{array}$ \\
\hline 3 & 40.3 & & 36.1 & 41.4 & 40.4 & 42.6 \\
\hline 4 & 30 & 92.8 & 26.3 & 31.7 & 29.6 & 34.9 \\
\hline 5 & 23.7 & & 20.7 & 25.4 & 23.4 & 30.2 \\
\hline 6 & 19.4 & 108.9 & 17.1 & 21.3 & 19.3 & 27 \\
\hline 7 & 16.4 & & 14.6 & 18.3 & 16.5 & 24.7 \\
\hline 8 & 14.2 & 118.5 & 12.7 & 16 & 14.4 & 22.9 \\
\hline 9 & 12.5 & & 11.3 & 14.2 & 12.7 & 21.4 \\
\hline 10 & 11.1 & 124.9 & 10.2 & 12.8 & 11.4 & 20.2 \\
\hline 11 & 10.1 & & 9.2 & 11.7 & 10.4 & 19.1 \\
\hline 12 & 9.1 & 129.9 & 8.4 & 10.7 & 9.5 & 18 \\
\hline
\end{tabular}

\section{SECTORS OF THE BASE TRANCEIVER STATION}

We will consider, as an example, the total number of elements for the BTS is 48 elements. The number of sectors may be any number and each sector may be covered by number of beams depending on the number of elements per sector and amplitude distribution (weight) of the elements as shown in Table 1. The BTS configuration will be considered with two options of 3 and 4 sectors as shown in Figure 1.The assumed sector angles of both sectors and direction of heavy and low traffic are given in Table 2. In 3-sector design, 16 elements are used per one sector with combinations of 4, 6, 8, and 12 elements to form a 16 elements array. There are a total of 5 options of array grouping for 16 elements as given in Table 3. In 4-sector design, 12 elements are used per one sector with combinations of $3,4,6$, and 8 elements. There are a total of 6 options of array grouping for 12 elements as given in Table 3. Individual beam widths produced by the grouped elements add up to form the sector beam width. Depending on the beam width required for each 3 and 4 sector, the array elements are connected into groups of $3,4,6$, 8 , or 12 configurations accordingly with specific amplitude distribution (weight) applied. The composition of 16 and 12 elements are arranged in a linear form along the radial direction. Individual beam widths produced by the grouped elements add up to form the sector beam width required according to the assigned traffic capacity.

Table 2: Assigned angles, in degrees, and direction of heavy and low traffic for the 3 and 4 sectors

\begin{tabular}{|l|l|l|l|l|l|l|}
\hline & $\begin{array}{l}\text { angle of } \\
\text { sector (1) }\end{array}$ & $\begin{array}{l}\text { angle of } \\
\text { sector (2) }\end{array}$ & $\begin{array}{l}\text { angle of } \\
\text { sector (3) }\end{array}$ & $\begin{array}{l}\text { angle of } \\
\text { sector (4) }\end{array}$ & $\begin{array}{l}\text { direction of } \\
\text { heavy traffic }\end{array}$ & $\begin{array}{l}\text { direction of } \\
\text { low traffic }\end{array}$ \\
\hline 3-sector & 100 & 185 & 75 & - & 12 o'clock & 10 o'clock \\
\hline 4-sector & 40 & 90 & 190 & 40 & 12 o'clock & 4 o'clock \\
\hline
\end{tabular}


Table 3: The possible combinations (options) of array grouping for 16 and 12 elements

\begin{tabular}{|l|l|l|l|l|l|l|}
\hline & option (1) & option (2) & option (3) & option (4) & option (5) & option (6) \\
\hline $\begin{array}{l}16 \\
\text { elements }\end{array}$ & 88 & 412 & 466 & 448 & 4444 & ---- \\
\hline $\begin{array}{l}12 \\
\text { elements }\end{array}$ & 12 & 66 & 48 & 444 & 336 & 3333 \\
\hline
\end{tabular}

\section{STRUCTURE OF THE PROPOSED GENETIC ALGORITHM}

Any GA starts with a population of randomly generated solutions [12], chromosomes, and advances toward better solutions by applying genetic operators modeled on the genetic processes occurring in nature. In these algorithms we maintain a population of solutions for a given problem; this population undergoes evolution in the form of natural selection. In each generation, relatively good solutions reproduce to give offspring that replace the relatively bad solutions which die. An evaluation or fitness function plays the role of environment to distinguish between good and bad solutions. In this part an adaptive GA is introduced and used to optimize the problem of dynamic sector synthesis of antenna array. The main parts of the proposed algorithm are described below.

\subsection{Population Initialization}

The implemented GA initializes the data members of the population and generates the first generation of chromosomes with random values. It uses the pure randomly initialization strategy which is preferable in research, because it clarifies the robustness of the algorithm. Initialization is done by randomly selecting items from the output of the "possible combination" function to represent what is called the initial population P or POP (0) where $\mathrm{P}$ is known as the population size.

\subsection{Evaluation Function}

The objective is to achieve each sector angle with suitable array combinations with minimum error. Three different error functions have been used.

The first one labeled as absolute error $\left(E_{a}(i)\right)$

which equals the difference between the required angle and the generated one obtained from the algorithm:
Where: $R(j)$ : Required angle of the sector $j$ (the output of sectoring function) A (i): Individual I (initially the output of possible combination function) During trials, the GA was based on the above error function. It has been found that some error values were large; which makes the average fitness converges to a suboptimal solution very fast. So the second suggested error function was labeled as

absolute normalized error $\left(E_{n}(i)\right)$ which equals

$\left(E_{a}(i)\right)$ divided by $\mathrm{R}(\mathrm{j}): R(j)-A(i)$

$E(i)=n R(j)$ The solution was improved and became closer to the optimum. The third error function was proposed as the squared absolute normalized error:

$\begin{aligned} 2 J_{E_{s}} & =R(j)-A(i) \\ \mid & R(j) \mid\rfloor\end{aligned}$

The fitness function is inversely proportional to the error function. So, the fitness function $F$ (i) is defined as: F (i) = 1/ (1+ E (i)), where E (i) is the selected error function. The reproduction operator method is the "biased roulette wheel" [6].

\subsection{Chromosome Crossover Operator}

The crossover operator is the element that provides the GA function that equivalent to the production of hybrids by genetics crossover in natural system. The simplest crossover operator takes bits from each parent and combines them to create child strings. The algorithm handles one type of crossover technique, one point crossover. Since our problem requires fine tuning of available values, we haven't used other crossover techniques, such as (two-point crossover). In one point crossover a random point is selected which divides each parent vector into two groups of genes. Then each child inherits one genes group 
from each parent successively. The crossover probability $\rho_{c}$ is defined as the probability or the

rate of cross-over along the population. This means that not all the selected parents are subjected to crossover process, but according to the probability test.

\subsection{Mutation Operator}

In this work a new mutation mechanism is introduced in which the mutation probability (Pm) is adaptive with a single chromosome as follows: $\mathrm{Pm}=0.1(1-\mathfrak{R})$. Where $\mathfrak{R}$ an adaptive parameter is varies between 0 and 1 . When $\Re$ is very small, the mutation probability is approximately 0.1 . As the value of $\mathfrak{R}$ increases, the new mutation

\subsection{Population Replacement Function}

This function integrates all GA operators to produce new generation of individuals. It loops to generate an equal number of children to the population size. In each loop it uses the selection function twice to select two parents from the current generation, and then generates the new

\section{SIMULATION RESULTS}

The algorithm has been tested for different GA parameters and the best combinations are: $\bullet \rho=0.9 c$

$$
\begin{aligned}
& \rho_{m}=\text { Adaptive probability }(0 \sim 0.1) \\
& \text { Pop-size }=(50 \sim 200)
\end{aligned}
$$

- Number of generations $=1000$ The simulation was done for the two configurations of the BTS with 3 and 4-sector shown in Figure 1. The simulation was done by using only two amplitude distributions weight-1, and weigt-2 (see Table 1) due to limitation of the computer memory size, but actual system will implemented to manipulate more than 10 weights. The results obtained using the three evaluation function, simple genetic algorithm (SGA) with constant mutation probability for the normalized and squared normalized error functions and the adaptive mutation probability algorithm (AGA) with squared normalized error. Results of the 3 sector configuration with different beam widths are given in Figures 2 and 3 for the SGA with squared normalized error and AGA. Figure 4 shows the average fitness for the 3-sector verses the number of generation for the three evaluation probability value decreases. Finally, when $\mathfrak{R}$ approaches unit, the mutation probability is zero and the new solution is the old one. Experiments were conducted with different values of $\mathfrak{R}$ and we have found that the optimal value of $\mathfrak{R}$ is the average fitness of the population. At first generations, the average fitness value is low as we are far from the optimal solution. Thus, the adaptive mutation provides the search space with new solution elements. The average fitness is improved as the GA successfully moves towards a better solution. At last, the average fitness approaches the best fitness which is approximately unity and the adaptive mutation operator maintains the best solution elements.

children that follow the problem constraints. By the end of this function we add the newly generated individuals to the population. When the population finishes its process of creating new individuals and then selects the best group of individuals to survive to the next generation. This strategy is called the rank replacement technique [6].

function. The solutions obtained from AGA are of higher fitness than SGA for the three sectors. Results of the 4-sector configuration with different beam widths are given in Figures 5 and 6 for the SGA with squared normalized error and AGA. Figure 7 shows the average fitness for the 4-sector verses the number of generation for the three evaluation function. The solutions obtained from AGA are of higher fitness than SGA for the four sectors. Results of both techniques (SGA and AGA) have narrow beam widths in heavy handoff areas (hot spots) and wider beam widths in areas with low traffic density. Comparison between the communication soft hand-off process. Effect of solution parameters for the 3 and 4-sector for the number of sectors on the convergence time has two different used techniques are given in Table 4 been studied. It is noted that the convergence times and 5 respectively. Total coverage angle should be are at 415, 94, and 20 generations for the 3, 4 and equal to $360^{\circ}$. The coverage angle error value is 6 sectors respectively. Figure 8 shows that the required to be zero or small positive value. convergence time of the AGA technique is Negative error value means creating a dead zone, inversely proportional to the number of designed while positive error value means creating an 
sectors. overlapping between beams which simplifies the

Table 4: Solution parameters of the 3- sector for the two different used techniques

\begin{tabular}{|l|l|l|l|}
\hline Used technique & average fitness & Convergence time & coverage angle error \\
\hline SGA & 1 & 460 generation & $-0.3 \%$ \\
\hline AGA & 1 & 415 generation & $+2 \%$ \\
\hline
\end{tabular}

Table 5: Solution parameters of the 4- sector for the two different used techniques

\begin{tabular}{|l|l|l|l|}
\hline Used technique & average fitness & Convergence time & coverage angle error \\
\hline SGA & 1 & 125 generation & $+2.2 \%$ \\
\hline AGA & 1 & 94 generation & $+0.7 \%$ \\
\hline
\end{tabular}

\section{COCLUSION}

This paper has met the objectives of investigating and analyzing the method of dynamic sector synthesis of linear arrays using genetic algorithm for the purpose of load balancing among the sectors of the BTS. The designs were further oriented to different heavy and low traffic density for load balancing. Successful configurations of

\section{REFERENCES}

[1] R. H. Roy, "An overview of smart antenna technology: The next wave in wireless communications," Proc. 1998 IEEE Aerospace Conf., vol. 3, May 1998, pp. 339-345.

[2] J.C. Liberti Jr. and T.S. Rappaport: Smart Antennas for Wireless Communications, Prentice Hall, Upper Saddle River, New Jersey, 1999.

[3] J. Lu and T. Ohira, "Smart antennas at wireless mobile computer terminals and mobile stations," Proc. 2001 IEEE AP-S Int. Symp. USNC/URSI Nat. Radio Sci. Meeting, Boston, July 8-13, 2001.

[4] Lee, H. F., and W. Chen, Advances in Microstrip and Printed Ant., New York, J. Wiley \& Sons, 1997.

[5] Ho, M-J., Stuber, G.L., Austin, M.D.,

[10] C. A. Balanis, Antenna Theory: analysis and [12] M. Gen and R. Cheng: Genetic Algorithms Design, 2nd Edition, John Wiley and Sons, and Engineering Design, John Wiley \& Sons, New York. Inc. New the sectors can be achieved based on traffic changes. A proposed adaptive GA is introduced and used to solve the considered problem. It has been shown that the SGA and AGA techniques were valuable and useful in solving the problem of dynamic sector synthesis of antenna array. The solution obtained using AGA is faster in convergence and has higher fitness than SGA

"Performance of Switched-Beam Smart Antennas for Cellular Radio Systems", IEEE Trans. on Vehicular Technology, Vol. 47, No. 1, pp. 10-19, February 1998

[6] Davis Lwrence, "Hand Book of Genetic Algorithm" Library of Congress Catalogy, USA, 1991.

[7] David E. Goldberg: Genetic Algorithm in search, Optimization, and machine learning. Addison-Wesley Publishing company, Inc, 1989.

[8] J. Jin, H. Wang, W. Zhu, and Y. Liu, "Array Patterns Synthesizing Using Genetic Algorithm", PIERS Online, Vol. 2, No. 1, 6468, 2006.

[9] R. S. Yahya and M. Eric: Electronic optimization by Genetic Algorithms, John Wiley, New York, 1999.

York,1997.

[11] D. M. Pozar, PCAAD 4: Personal Computer Aided Antenna Design, Antenna Design Associates, Leverett, MA,1999. 


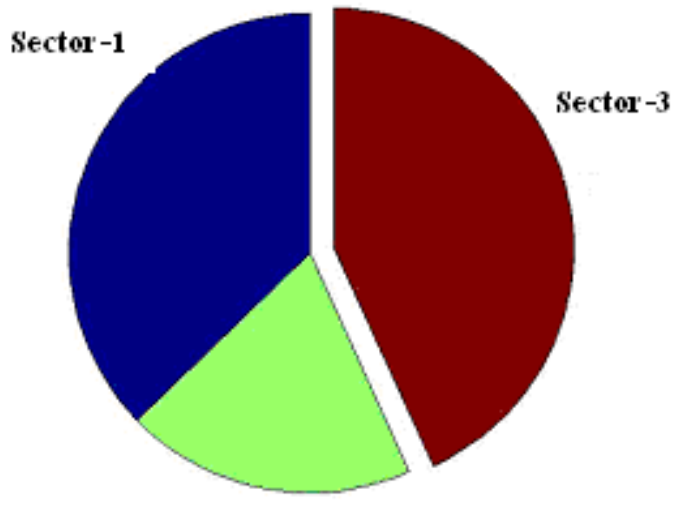

Sector -2

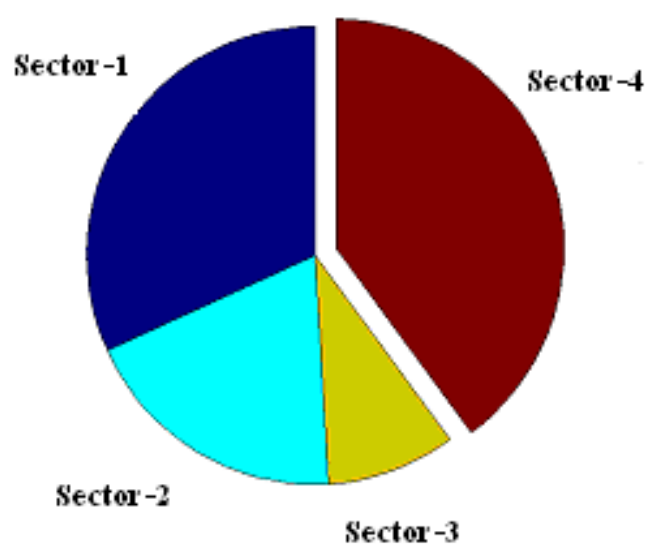

Sector -3

Figure 1: Configuration of the BTS with 3 and 4sectors
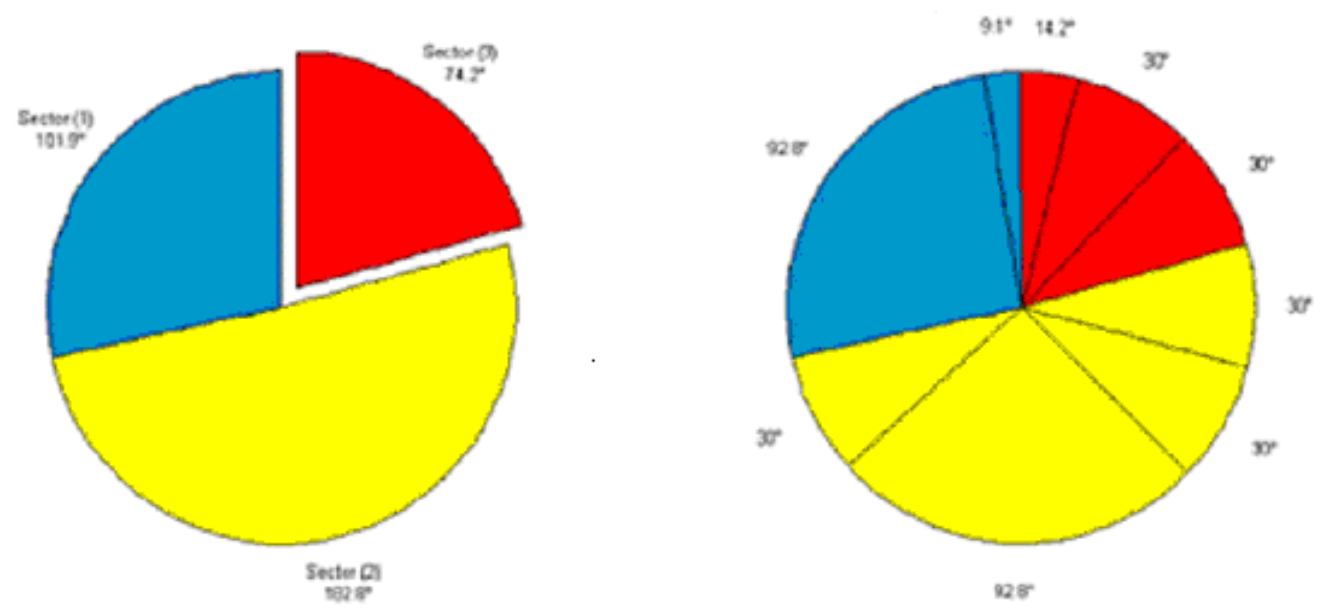

Cover ed angle $=358.9$

Figure 2: 3-sector configuration results from the SGA technique 

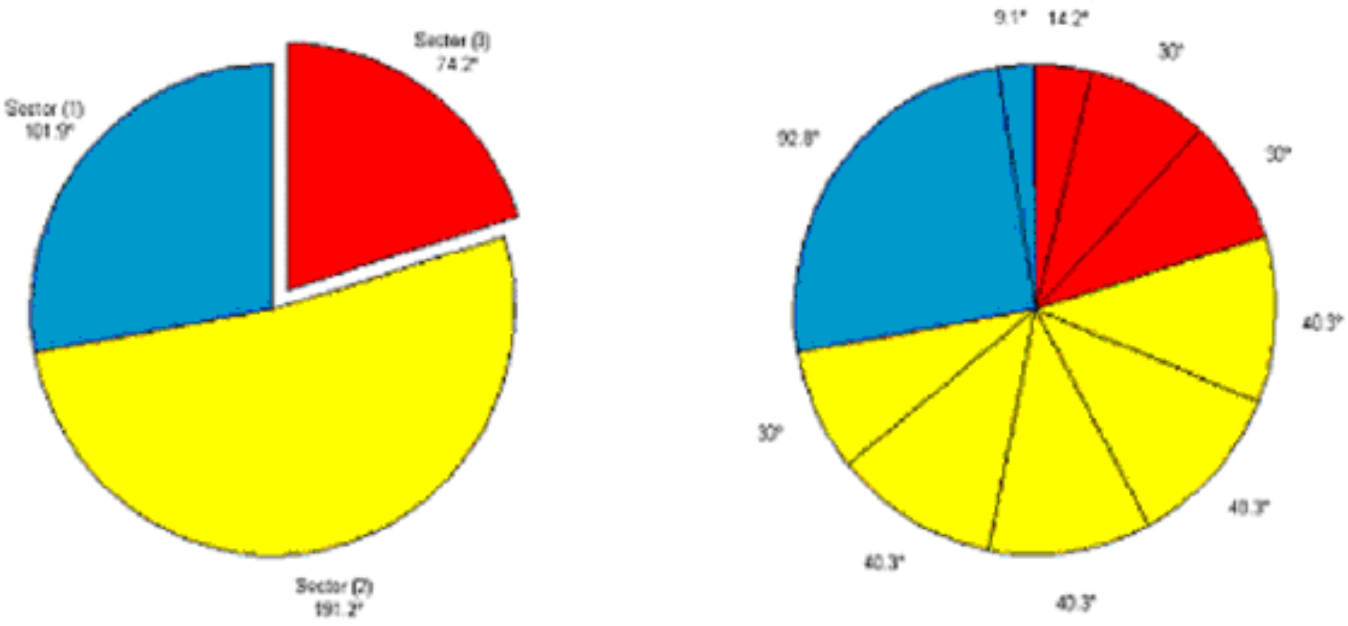

Cover ed angle $=367.3$

Figure 3: 3-sector configuration results from the AGA technique

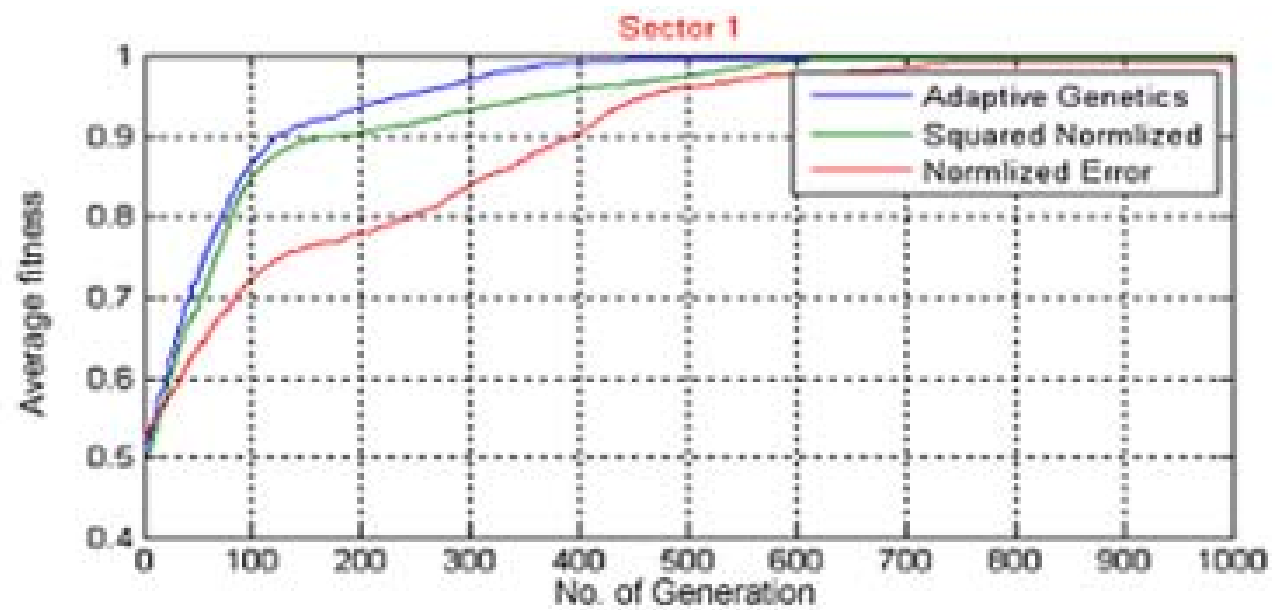

Sector 2

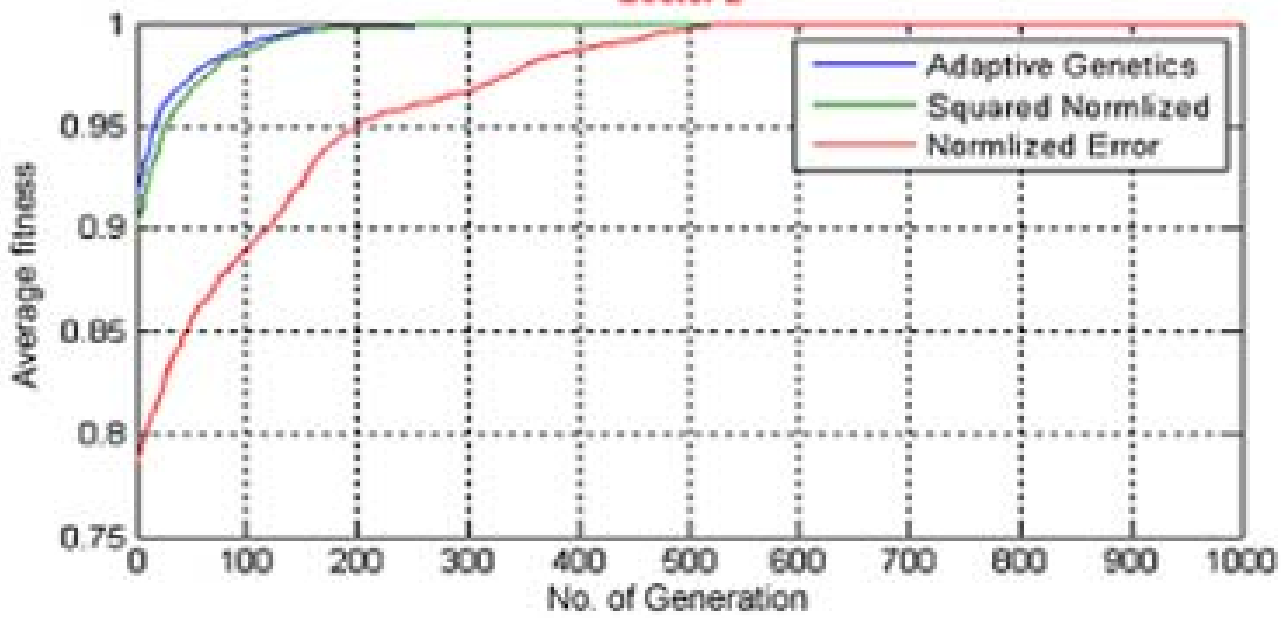




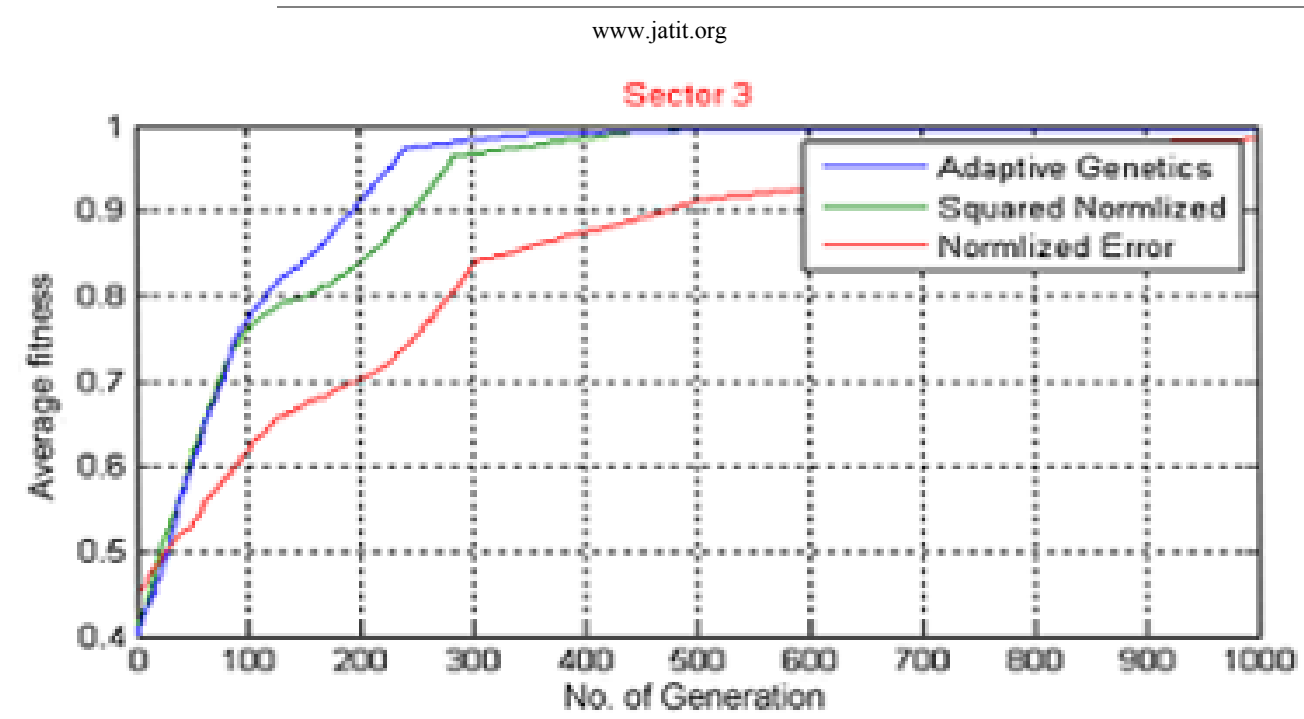

Figure 4: Average fitness for the 3-sector vs. the number of generation for the three evaluation function
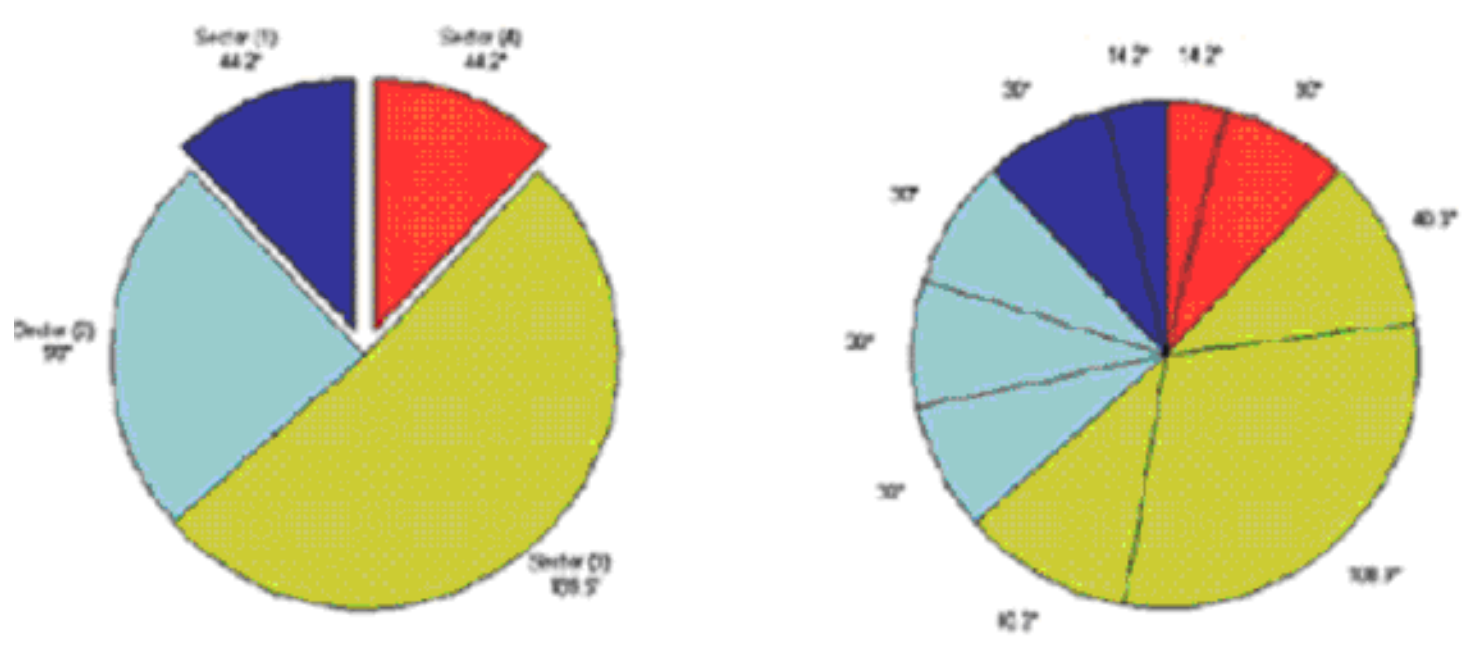

Cover ed angle $=367.9$

Figure 5: 4-sector configuration results from the SGA technique 
www.jatit.org
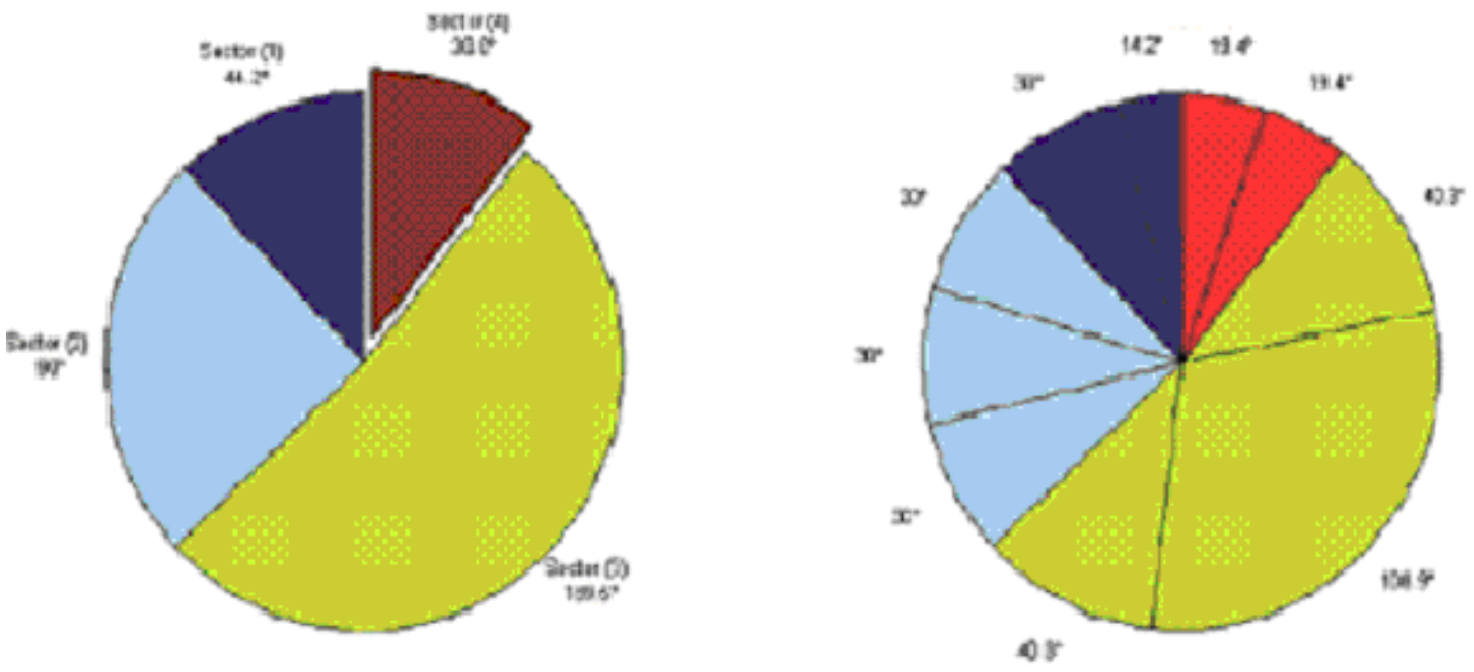

Cover ed angle $=362.5$

Figure 6: 4-sector configuration results from the AGA technique
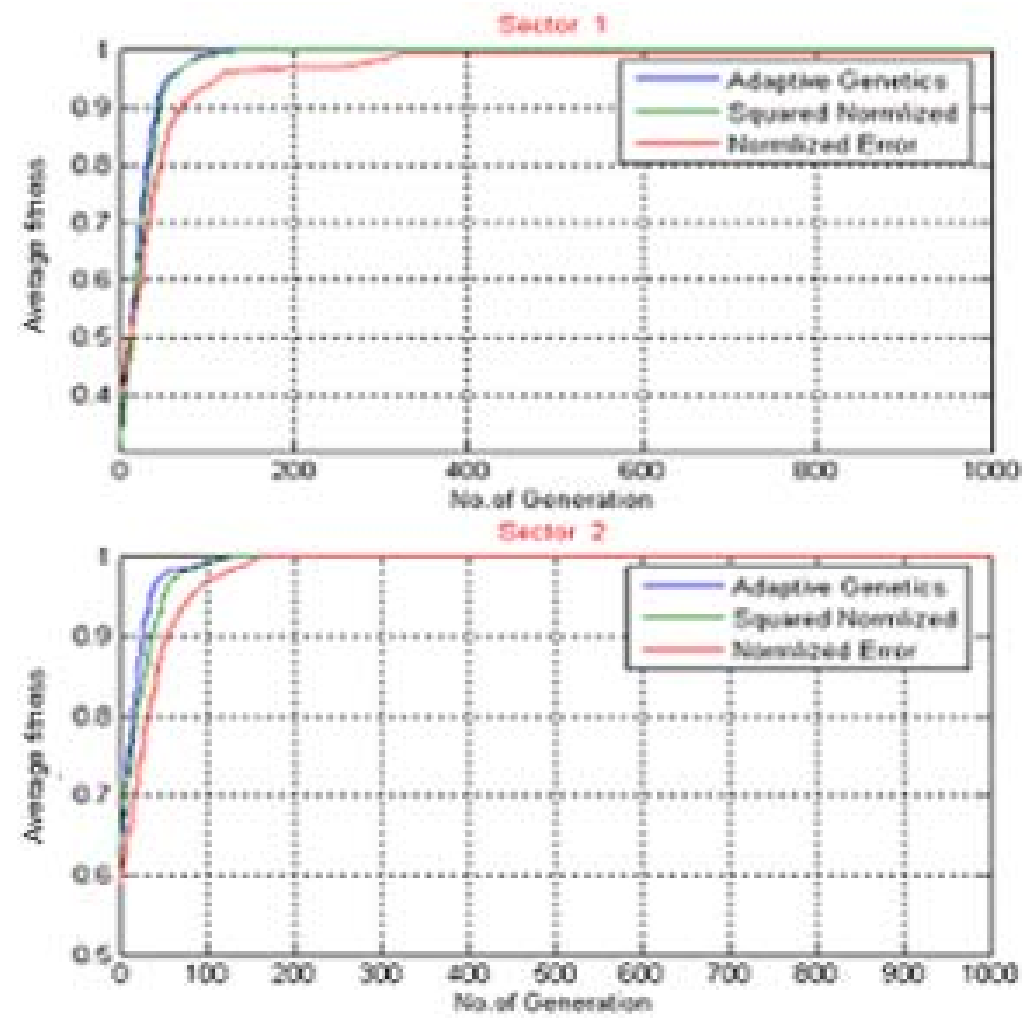

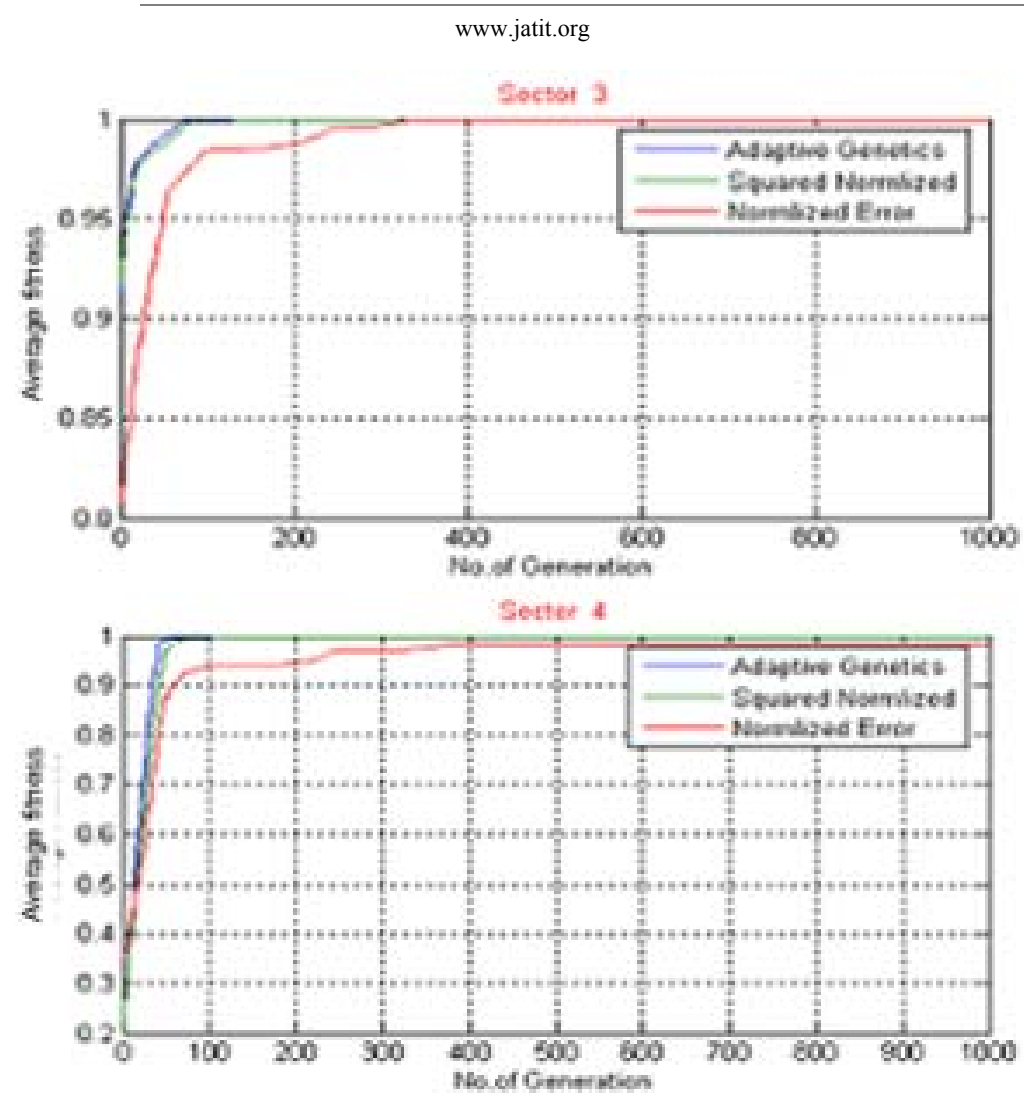

Figure 7: Average fitness for the 4-sector vs. the number of generation for the three evaluation function

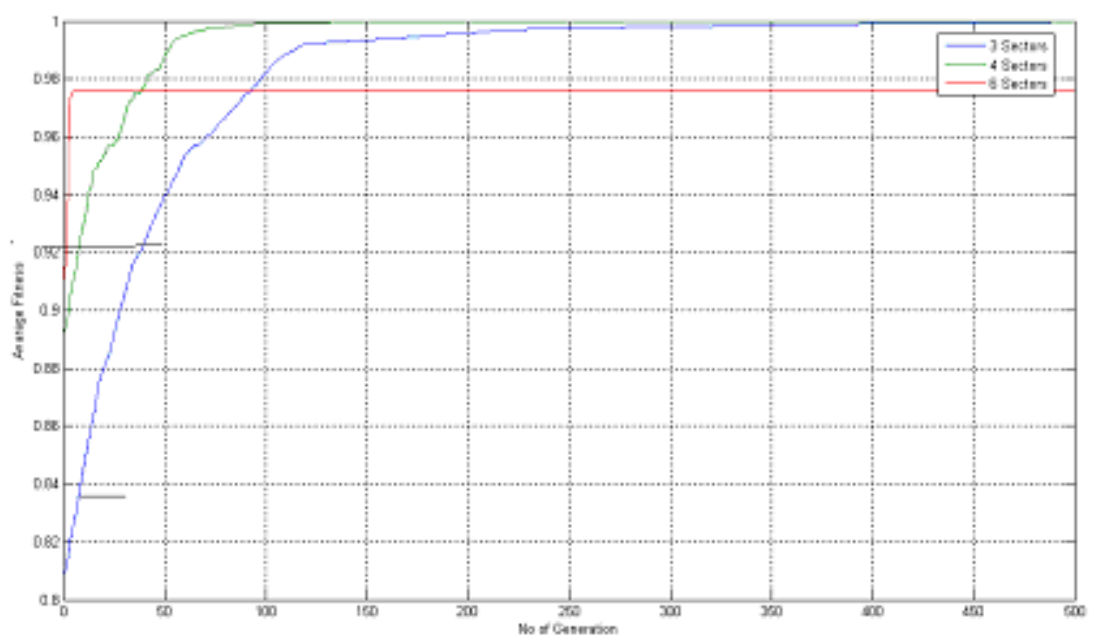

Figure 8: Average fitness vs. number of generation adaptive genetic algorithm for the 3, 4 and 6 sectors 